\title{
QUALITY IMPROVEMENT CURRICULAR CONTENT IN MEDICAL EDUCATION IN THE UNITED STATES
}

\author{
Jason White, BS, MBA \\ Medical Student, Indiana University School of Medicine \\ jawwhite@iupui.edu \\ Deanna R. Willis, MD, MBA, FAAFP \\ Associate Professor of Family Medicine, Indiana University School of Medicine \\ drwillis@iupui.edu
}

\begin{abstract}
:
The need to include quality improvement curricula into formal medical education in the United States has been identified and publicized for many years. However, despite awareness of the need for medical learner acquisition of knowledge, skills, and attitudes in the area of quality improvement, there is wide variation in the type and content of curricular material and experiences. Gaps include perceived lack of curricular time, lack of faculty development, organizational barriers, and lack of clinical outcomes data. Research to better understand the gaps, identify solutions to address the gaps, and evidence of improved clinical outcomes are necessary to achieve the level of workforce training needed for developing models of healthcare delivery such as value based care and population health.
\end{abstract}

Key words: Quality Improvement, Medical Education, Learner, Practice Based Learning and Improvement, Systems Based Care, Value Based Care, Population Health 


\section{SECTION 1.1 BACKGROUND}

Since the late 1990s, with the publishing of the Institute of Medicine's reports To Err is Human: Building a Safer Health System and Crossing the Quality Chasm: A New Health System for the 21 $1^{\text {st }}$ Century, there has been an increasing recognition that quality improvement (QI) should be included in some form in the undergraduate and graduate medical education curriculum, to more fully prepare medical students and residents for their future careers as physicians. ${ }^{5}$ The Association of American Medical Colleges, the Accreditation Council for Graduate Medical Education, and the World Health Organization have all created guidelines and strategies to enable teaching institutions at the Undergraduate Medical Education (UME) and Graduate Medical Education (GME) level to include QI in their curriculum. ${ }^{2,} 8$ Many innovations in curricular form and content have been created by various schools, ranging from a classroom lecture series in the pre-clinical years to full involvement of students and residents in QI projects at clinical sites. ${ }^{10}$ At this time, however, there is limited information as to the ultimate success of all these endeavors; will the next generation of physicians be active participants in QI during their professional careers, or will they regard it as an interesting, but ultimately unused, part of the medical education?

\section{SECTION 1.2 TYPES OF QUALITY IMPROVEMENT CURRICULA}

Despite the call by national governing bodies, such as the Accreditation Council for Graduate Medical Education
(ACGME) and the Liaison Committee on Medical Education (LCME), to include QI curriculum at the undergraduate and graduate levels, students' knowledge of QI methodologies is low, though understanding increases with exposure through curriculum. ${ }^{2}$ Many students report that their instruction in QI is inadequate during their undergraduate medical education. ${ }^{10,11}$ When the curriculum is included, the success can vary based on the format of the curriculum. Projects involving solely chart-audits left many students feeling left out of the change process. ${ }^{3}$ Many students report that hands-on experience in QI in a clinical setting, being led by practicing physicians, provides superior learning to didactic or small groupbased formats. ${ }^{5}$

\section{SECTION 1.3 LEARNER INTEREST IN QUALITY IMPROVEMENT CURRICULAR CONTENT}

Learner interest in QI is largely unknown at this point. Many surveys have evidenced that students want increased exposure to QI in their training. 5,10 The majority of students believe that such training is at least equal to, or sometimes greater in importance, than their basic science and clinical training. ${ }^{10}$ Little data exists, however, to determine the students' interest in continuing to pursue QI work beyond their required coursework. Residents often express interest in continuing to work toward change goals set during QI training, but only close to half of those goals are actually seen through to the end. ${ }^{6}$ There is a severe lacking of information regarding whether or not the students receiving training in QI methodology will continue to use it after their training period has ended. 
Inclusion of QI methodologies and experience in medical student curriculum can not only result in better learning outcomes, but can often produce tangible benefits in patient care. ${ }^{4,13}$ Multiple studies have shown that educators believe that students and residents should be involved in QI efforts and that the involvement of the learners will improve the projects' outcomes. ${ }^{12}$ Additionally, residents believe that being introduced to QI work early in a post-graduate medical career can lead to better integration into a work ethic and practice habits. ${ }^{6}$ Graduating medical students also believe that inclusion of QI curriculum in their training results in them being more adequately prepared them for their work as residents and as practicing physicians. ${ }^{5}$

\section{SECTION 1.4 GAPS IN QUALITY IMPROVEMENT CURRICULA}

There are many gaps between the current state and future state, where QI is fully integrated into curriculum and builds lasting habits and interest in the student and resident learners. One large barrier is the perception that many medical students do not have sufficient time to focus on hands-on learning doing a full QI project. ${ }^{12}$ Attaching the project to a year-long clerkship overcomes this barrier, ${ }^{7}$ but such a format is not a possibility at many medical schools. Another large challenge is overcoming organizational cultures in the teaching hospitals which do not promote the value of QI work. ${ }^{8}$ Creating organizational change is not easy, and some success have been made through patience, starting with small projects, and bringing in many different stakeholders to create buy-in across the organization. ${ }^{1}$
Additionally, though QI milestones are mandatory as a part of the ACGME requirements, many residents view the work as something done simply to "meet a requirement" rather than a means to build professional skills which will last a lifetime. ${ }^{9}$ This perception can stem from faculty's discomfort with QI topics and subsequent failure to practice QI themselves as well as the tendency to limit QI work to residents' individual projects, rather than involving them in system-wide initiatives. ${ }^{8}$

Finally, there is little to no information to determine if medical students and residents will commit the training they receive from curricular training to heart and use it in the future. Moving into the future, increased efforts must be made to overcome some of these gaps, and specifically efforts should be made to further understand learners' interest in QI philosophies and methodologies and their willingness to commit to doing QI throughout their careers as physicians.

\section{SECTION 1.5 FUTURE DIRECTIONS}

Growing the body of evidence regarding the types and impact of QI curricula on patient or population based clinical outcomes is essential based on limited resources for medical education at both the UME and GME level. With the majority of QI curricular experiences being didactic in nature, ${ }^{12}$ identifying and supporting robust methods to link educational models to clinical outcomes is necessary.

Research is needed to understand factors that are related to the presence of QI curricula, particularly curricula that are linked to improved clinical outcomes. While 
educators who believe that QI is important and that learners should achieve competency in QI knowledge and skills are more likely to build QI curricula, ${ }^{12}$ little is known about what influences the beliefs of educators on this topic.

The keys to this final success, in the authors' opinions, are the presence of experiential and meaningful QI curricular content throughout the UME and GME years, piquing the interest of students and residents in QI methodology, and establishing evidence that introducing QI curriculum into medical school education leads to better learning outcomes and patient outcomes. 


\section{RESOURCES}

1. Blanchard RD, Pierce-boggs K, Visintainer PF, Hinchey KT. Integrating Quality Improvement With Graduate Medical Education: Lessons Learned From the AIAMC National Initiatives. Am J Med Qual. 2015;

2. Blasiak RC, Stokes CL, Meyerhoff KL, Hines RE, Wilson LA, Viera AJ. A cross-sectional study of medical students' knowledge of patient safety and quality improvement. N C Med J. 2014;75(1):15-20.

3. Gould BE. Adding continuous quality improvement to a medical school curriculum: problems and possibilities. Virtual Mentor. 2004;6(6)

4. Gould BE, Grey MR, Huntington $\mathrm{CG}$, et al. Improving patient care outcomes by teaching quality improvement to medical students in community-based practices. Acad Med. 2002;77(10):1011-8.

5. Gould BE, O'Connell MT, Russell MT, Pipas CF, Mccurdy FA. Teaching quality measurement and improvement, costeffectiveness, and patient satisfaction in undergraduate medical education: the UME21 experience. Fam Med. 2004;36 Suppl:S57-62.

6. Jansma JD, Wagner C, Bijnen AB. Residents' intentions and actions after patient safety education. BMC Health Serv Res. 2010;10:350.

7. Levitt DS, Hauer KE, Poncelet A, Mookherjee S. An innovative quality improvement curriculum for third-year medical students. Med Educ Online. 2012;17

8. Myers JS, Nash DB. Graduate medical education's new focus on resident engagement in quality and safety: will it transform the culture of teaching hospitals?. Acad Med. 2014;89(10):1328-30.

9. Nasca TJ, Weiss KB, Bagian JP. Improving clinical learning environments for tomorrow's physicians. $\mathrm{N}$ Engl $\mathrm{J}$ Med. 2014;370(11):991-3.

10. Teigland CL, Blasiak RC, Wilson LA, Hines RE, Meyerhoff KL, Viera AJ. Patient safety and quality improvement education: a cross-sectional study of medical students' preferences and attitudes. BMC Med Educ. 2013;13:16.

11. Varkey P. Educating to improve patient care: integrating quality improvement into a medical school curriculum. Am J Med Qual. 2007;22(2):112-6.

12. Willis DR, Bennett I, Jones BG, Renshaw SE, Holley M, Dankoski ME. Practice-based learning and improvement in family medicine student clerkships: a CERA study. Fam Med. 2014;46(6):423-8.

13. Wong BM, Etchells EE, Kuper A, Levinson W, Shojania KG. Teaching quality improvement and patient safety to trainees: a systematic review. Acad Med. 2010;85(9):1425-39. 